\title{
Effect of VEGF-C siRNA and endostatin on ring formation and proliferation of esophageal squamous cell carcinoma lymphatic endothelial cells
}

This article was published in the following Dove Press journal:

OncoTargets and Therapy

31 October 2016

Number of times this article has been viewed

\author{
Yuping Zheng ${ }^{1-3, *}$ \\ Miaomiao Sun ${ }^{4, *}$ \\ Jinyan Chen ${ }^{1,2}$ \\ Lulu $\mathrm{He}^{1,2}$ \\ $\mathrm{Na} Z$ hao ${ }^{1,2}$ \\ Kuisheng Chen ${ }^{1,2}$ \\ 'Pathology Department, The First \\ Affiliated Hospital of Zhengzhou \\ University, ${ }^{2} \mathrm{Henan}$ Key Laboratory \\ of Tumor Pathology, ${ }^{3}$ Pathology \\ Department, The Second Hospital \\ of Shandong University, Jinan, \\ ${ }^{4}$ Pathology Department, Henan \\ Tumor Hospital, Zhengzhou, \\ People's Republic of China \\ *These authors contributed equally \\ to this work
}

Objective: To study the effects of vascular endothelial growth factor $\mathrm{C}$ small interfering RNA and endostatin on esophageal squamous cell carcinoma-related ring formation in vitro and proliferation of lymphatic endothelial cells.

Materials and methods: KYSE150 cells were subjected to analysis of cell transfection and endostatin operation. The groups were as follows: negative group, blank group, negative plus endostatin group, endostatin group, SG1 group, SG2 group, SG1 plus endostatin group, and SG2 plus endostatin group. The esophageal cancer-related microlymphatic endothelial cells were three-dimensionally cultured. Cell Counting Kit-8 (CCK-8) assay was employed to detect cell proliferation.

Results: The negative group's three-dimensional culture result was the highest, followed by the blank group, negative plus endostatin group, endostatin group, SG2 group, SG1 group, SG1 plus endostatin group, and SG2 plus endostatin group. The quantity of living cells in the blank group was the highest, followed by the negative control, endostatin, SG2, SG1, negative plus endostatin, SG1 plus endostatin, and SG2 plus endostatin groups.

Conclusion: Both vascular endothelial growth factor $\mathrm{C}$ small interfering RNA and endostatin could inhibit ring formation in esophageal squamous cell carcinoma and proliferation of lymphatic endothelial cells.

Keywords: esophageal squamous carcinoma cells, esophageal cancer-associated lymphatic endothelial cells, VEGF-C, ring formation, proliferation

\section{Introduction}

Metastasis by lymphatic route is one of the main pathways for the spread of esophageal cancer and also one of the main reasons for postoperative recurrence of esophageal cancer. Vascular endothelial growth factor C (VEGF-C) is one of the main factors that regulates lymphatic genesis and is highly expressed in many tumor tissues, which can specifically work on the lymphatic endothelial cells, stimulate the proliferation of the lymphatic epithelial cells, and induce lymphatic genesis. ${ }^{1}$

Endostatin is a naturally occurring $20-\mathrm{kDa}$ C-terminal fragment derived from type XVIII collagen. It is reported to serve as an antiangiogenic agent, similar to angiostatin and thrombospondin. Endostatin is a broad spectrum angiogenesis inhibitor and may interfere with the proangiogenic action of growth factors such as basic fibroblast growth factor and VEGF. Endostatin may also be useful as a therapeutant inr inflammatory, diseases, such as rheumatoid arthritis as well as Crohn's disease, diabetic retinopathy, psoriasis, and endometriosis, for reducing the infiltration of inflammatory cells through invading angiogenesis. Down's syndrome patients seem to be protected from diabetic
Pathology Department, First Affiliated Hospital of Zhengzhou University, 100 Kexue Street, Gaoxin District, Zhengzhou 450052, Henan, People's Republic of China

Tel +86 37I 6778 3III

Email kuishengchen20I5@sina.com 
retinopathy due to an additional copy of chromosome 21 and elevated expression of endostatin.

Endostatin factor can effectively inhibit the proliferation of the lymphatic endothelial cells, thus inhibiting the formation of lymphatic vessels. ${ }^{2-4}$ In this study, we inactivated the VEGF-C expression of esophageal carcinoma cells and regulated esophageal cells through endostatin.

\section{Materials and methods}

Human esophageal cancer-related microlymphatic endothelial cells were purchased from Shanghai Bioleaf Biotech Co. Ltd. (Shanghai, People's Republic of China). Human poorly differentiated esophageal squamous carcinoma cell line KYSE150 cells were bought from Shanghai Bioleaf Biotech Co. Ltd.; Endothelial cell medium was purchased from Sciencell Research Laboratories (Carlsbad, CA, USA); Dulbecco's Modified Eagle's Medium and RM1640 medium were purchased from Beijing Solarbio Science \& Technology Co, Ltd. (Beijing, People's Republic of China); and Endothelin inhibitors were purchased from PeproTech (Rocky Hill, NJ, USA). Transfection reagent kit was purchased from GBI (Shanghai, People's Republic of China). Plasmids were bought from Genepharma (Shanghai, People's Republic of China). CCK-8 assay kit was purchased from Bogoo Biotechnology Co. Ltd (Shanghai, People's Republic of China). The design and synthesis of small interfering RNA (siRNA) were based on siRNA design principles and methods of Genepharma. Three siRNA sequences were designed and synthesized, as seen in Table 1.

Endostatin is a naturally occurring $20-\mathrm{kDa}$ C-terminal fragment derived from type XVIII collagen. It is reported to serve as antiangiogenic agent, similar to angiostatin and thrombospondin. Endostatin is a broad-spectrum angiogenesis inhibitor and may interfere with the proangiogenic action of growth factors such as basic fibroblast growth factor and VEGF.

Three-dimensional cultivated tube formation assay was performed as described previously. Wells of a 24-well plate were coated with Matrigel according to the manufacturer's instructions (Becton Dickinson) and incubated at $37^{\circ} \mathrm{C}$ for 30 minutes. Cells were grown in 6-well plates in the

Table I siRNA sequences

\begin{tabular}{lll}
\hline $\begin{array}{l}\text { Serial } \\
\text { number }\end{array}$ & siRNA & Sequence \\
\hline SG0 & VEGF-C-homo-579 & GGCTTATGCAAGCAAAGATCT \\
SGI & VEGF-C-homo-1056 & CCAATTACATGTGGAATAATC \\
SG2 & VEGF-C-homo-II66 & GCCGATGCATGTCTAAACTGG
\end{tabular}

Note: The sequences of siRNA in each group were demonstrated.

Abbreviation: siRNA, small interfering RNA. presence of DNAzymes $(0.3 \mu \mathrm{M})$ mixed with Lipofectamine $(5 \mu \mathrm{g} / \mathrm{mL})$ for 24 hours. Then they were detached with $1 \mathrm{mM}$ ethylenediaminetetraacetic acid, sedimented by centrifugation for 5 minutes, and resuspended in cell culture medium. Cells were added to Matrigel-coated wells and incubated for 24 hours.

According to procedures outlined by the Kamiya Biomedical Co. (Tukwila, WA, USA), CCK-8 assay (the kit of Cat No: KT-10150) was performed as described previously. $^{2-4}$

According to human VEGF-C, three interference sequences were designed, SG0, SG1, and SG2; the best interference sequence was selected based on the CCK-8 assay. During the endothelin inhibition test, different concentrations of endostatin were used to treat KYSE150 cells.

The blank control group was employed for the conditional medium of esophageal cancer cells and was not transfected. The negative control group was employed for the conditional medium of empty plasmid-transfected esophageal cancer cells. The SG1 group was employed for the conditional medium of SG1 plasmid-transfected esophageal cancer cells. The SG2 group was for the conditional medium of SG2 plasmid transfected esophageal cancer cells. The blank plus endostatin group was employed for esophageal cancer cells conditional medium processed with best concentration of endostatin. The negative plus endostatin group was employed for the conditional medium transfected with empty plasmid and endostatin processed. The SG1 plus endostatin group was employed for the conditional medium transfected with SG1 and endostatin processed. The SG2 plus endostatin group was employed for conditional medium transfected with SG2 and endostatin processed. The esophageal carcinoma-related microlymphatic endothelial cells were three-dimensionally cultivated, and the tubular structure formed by each group was calculated as well. CCK-8 assay was applied to detect cell proliferation.

\section{Statistical analysis}

SPSS 17.0 statistical software (SPSS Inc., Chicago, IL, USA) was employed to analyze the data. Measurement data were analyzed by one-way analysis of variance. Variance was used in comparison between different groups. The people counting the ring formation were blinded to the treatments. The counts were confirmed by three persons.

\section{Results \\ Plasmid screening and endostatin best inhibition concentration selection}

Figure 1 shows that the expression quantities of VEGF-C protein in KYSE150 cells of SG1 and SG2 groups were 


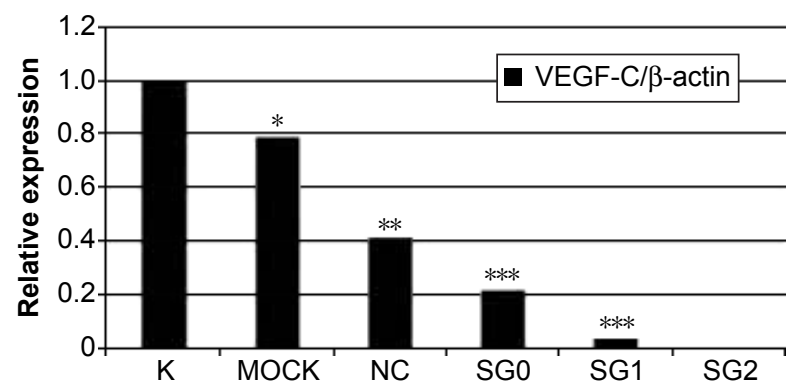

Figure I The relative expression of VEGF-C protein in KYSEI 50 cells.

Notes: The cells were treated as described in the "Materials and methods" section and then the cells were subjected to Western blot analysis. $\left({ }^{*} P<0.05\right.$, $* * P<0.01$, and $* * * P<0.00$ I; Student's $t$-test).

Abbreviations: K, positive control; MOCK, blank control; NC, negative control.

significantly lower than other groups after 96 hours of transfection. SG1 and SG2 groups had superior inhibitory effect than other groups. Therefore, SG1 and SG2 groups were selected for the downstream experiments.

Figure 2 shows that endostatin had the best inhibition effect on cancer cells when endostatin inhibition concentration was $12.8 \mu \mathrm{g} / \mathrm{mL}$. Therefore, the concentration of $12.8 \mu \mathrm{g} / \mathrm{mL}$ was chosen for the next downstream experiment.

\section{Effects of VEGF-C and endostatin on esophagel squamous cell carcinoma and clone formation}

Overall comparison showed that the quantity of living cells was highest in the negative control group, followed by the blank control, endostatin, negative plus endostatin, SG2, SG1, SG1 plus endostatin, and SG2 plus endostatin groups, among which there was no statistically significant difference between the groups $(P>0.05)$. There was no statistical significance found by comparing the two SG1 groups, SG2 groups, endostatin groups, and NC plus endostatin groups $(P>0.05)$. Comparison between SG1 plus endostatin and SG2 plus endostatin groups showed no statistical significance $(P>0.05)$. The comparison between each two of the

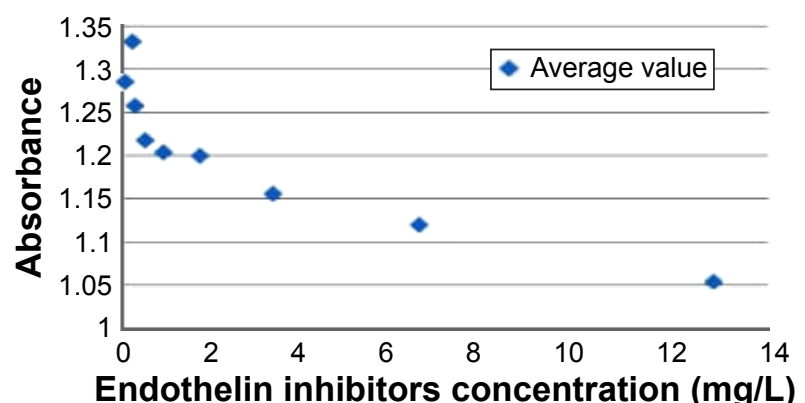

Figure 2 Effects of different concentrations of endostatin on the proliferation of KYSEI 50 cells.

Notes: The cells were treated as described in the "Materials and methods" section and then the average absorbance value was analyzed.
Table 2 Ring formation quantity of the three-dimensional cultivated KYSEI 50 cells

\begin{tabular}{|c|c|c|c|}
\hline \multirow[t]{2}{*}{ Group } & \multicolumn{3}{|l|}{ Time } \\
\hline & 3 days & 5 days & 7 days \\
\hline Blank control group & $32 \pm 1$ & $35 \pm 1.73$ & $33.67 \pm 1.16$ \\
\hline Negative control group & $30.67 \pm 1.16$ & $35.33 \pm 1.16$ & $33 \pm 2$ \\
\hline SGI group & $24 \pm 1.73$ & $26.67 \pm 0.58$ & $23.33 \pm 1.53$ \\
\hline SG2 group & $24 \pm 1$ & $28.33 \pm 0.58$ & $25 \pm 1$ \\
\hline Endostatin group & $26 \pm 1$ & $29.33 \pm 1.16$ & $28 \pm 1$ \\
\hline Negative plus endostatin group & $25 \pm 1$ & $28 \pm 1$ & $24.67 \pm 1.16$ \\
\hline SGI plus endostatin group & $17.67 \pm 1.16$ & $22 \pm 1$ & $21 \pm 1.73$ \\
\hline SG2 plus endostatin group & $17 \pm 1$ & $22 \pm 2$ & $20 \pm 1$ \\
\hline$F$ & 64.353 & 49.359 & 41.740 \\
\hline$P$-value & 0.000 & 0.000 & 0.000 \\
\hline
\end{tabular}

Notes: The cells were treated as described in "Materials and methods" section and then the cells were subjected to three-dimensional cultivated analysis. Data presented as mean $\pm S D$ or number.

remaining groups showed statistical significance $(P<0.05)$, as seen in Table 2, and Figures 3 and 4.

\section{The effect of VEGF-C siRNA and endostatin on esophageal squamous carcinoma lymphatic vessel endothelial cell proliferation in vitro}

The KYSE150 cells conditional medium was used in three-dimensional culture, and the cell proliferation condition of each group was low; the quantity of living cells in blank control group was the highest, followed by negative control, endostatin, negative plus endostatin, SG2, SG1 plus endostatin, and SG2 plus endostatin groups. There was no statistical significance between the groups $(P>0.05)$. There was no statistical significance between the two SG1 groups,

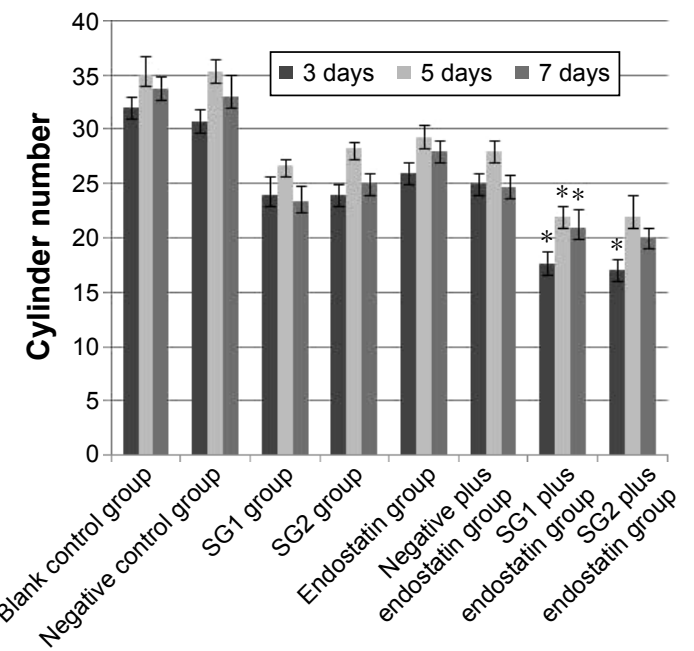

Figure 3 Ring formation quantity of the three-dimensional cultivated KYSEI 50 cells. Notes: The cells were treated as described in the "Materials and methods" section and then the cells were subjected to three-dimensional cultivated analysis. $* P<0.05$, Student's t-test. 

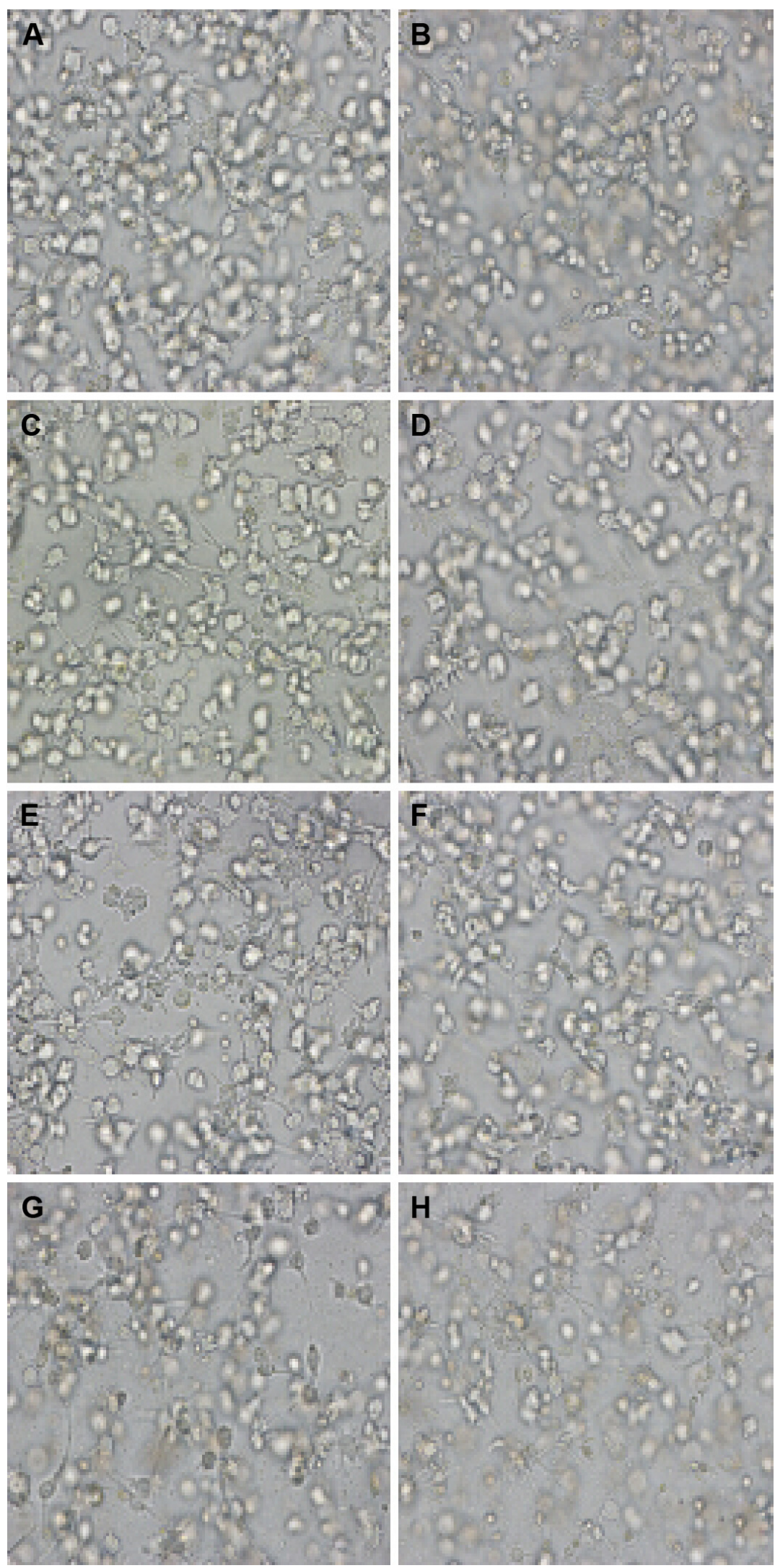

Figure 4 Ring formation situation of the three-dimensional cultivated KYSEI 50 cells (200x).

Notes: (A) Blank control group; (B) negative control group; (C) SGI group; (D) SG2 group; (E) endostatin group; (F) negative plus endostatin group; (G) SG I plus endostatin group; (H) SG2 plus endostatin group. The cells were treated as described in "Materials and methods" section and then the cells were subjected to three-dimensional cultivated analysis. 
Table 3 Proliferation of KYSEI50 cells under the threedimensional cultivated conditional medium

\begin{tabular}{lll}
\hline Group & $\begin{array}{l}\text { OD value, } \\
\text { mean } \pm \text { SD }\end{array}$ & Statistical items \\
\hline Blank control group & $2.566 \pm 0.028$ & \\
Negative control group & $2.53 \mathrm{I} \pm 0.023$ & \\
SGI group & $1.794 \pm 0.1 \mathrm{II}$ & $F=90.24 \mathrm{I}$ \\
$\mathrm{SG} 2$ group & $1.867 \pm 0.025$ & \\
Endostatin group & $1.923 \pm 0.106$ & \\
Negative plus endostatin group & $1.869 \pm 0.032$ & $P=0.000$ \\
SGI plus endostatin group & $1.566 \pm 0.00 \mathrm{I}$ & \\
SG2 plus endostatin group & $1.539 \pm 0.02$ & \\
\hline
\end{tabular}

Notes: The cells were treated as described in "Materials and methods" section and then the cells were subjected to CCK8 analysis.

Abbreviation: OD, optical density.

SG2 groups, endostatin groups, and NC plus endostatin groups $(P>0.05)$. There was no statistical significance found between SG1 plus endostatin and SG2 plus endostatin groups $(P>0.05)$. There were statistically significant differences between different groups $(P<0.05)$, as seen in Table 3 and Figure 5.

\section{Discussion}

In this study, VEGF-C and endostatin, the two thoroughly studied factors, were selected to observe their impact on inhibiting proliferation and metastasis of esophageal cancerrelated lymphatic vessel endothelial cells. In 1995, Hatva et al reported that VEGF-C or VEGF receptor-3 was the only discovered regulation factor that could regulate embryonic tissue lymphatic genesis and adjust mature individual lymphatic physiological function. ${ }^{5}$ Experiments confirmed that the expression of VEGF-C has a certain relation with tumor lymphatic metastasis, the overexpression of VEGF-C could stimulate tumor tissue lymphatic genesis. ${ }^{6-9}$ Hirakawa et al studied and discovered that VEGF-C could promote lymph node formation before the occurrence of tumor metastasis, then accelerate distant tumor metastasis through sentinel lymph node. ${ }^{10}$ Fukumoto et al reported that endostatin could inhibit tumor lymphatic genesis and lymph node metastasis through the downregulation of VEGF-C expression. ${ }^{11}$ From the experiment of Brideau et al, it was confirmed that the overexpression of endostatin could reduce the messenger RNA level of lymphatic vessel-related VEGF signal components, thus inhibiting tumor lymphatic vessel genesis and lymphatic metastasis. ${ }^{12}$

From the experimental results, it was found that the tube formation quantity of downregulated VEGF-C and endostatin processing groups were lower than that of blank control and negative control groups. Downregulation of esophageal cancer cells' VEGF-C expression and endostatin processing application could inhibit the related lymphatic endothelial cells tubing formation and proliferation, thus inhibiting lymphatic vessels genesis. At the same time, the downregulated VEGF-C group tube quantity was less than that of the endostatin processed group, which demonstrated that the function of downregulated VEGF-C on tube formation and proliferation of inhibiting lymphatic vessel endothelial cells was stronger than that of endostatins. The ring formation and cell proliferation quantity of synergistic inhibition of esophageal cancer-related lymphatic vessel endothelial cells was the least, which indicated the synergistic effect had the strongest effect on inhibiting lymphatic vessels endothelial cells tube formation and proliferation,

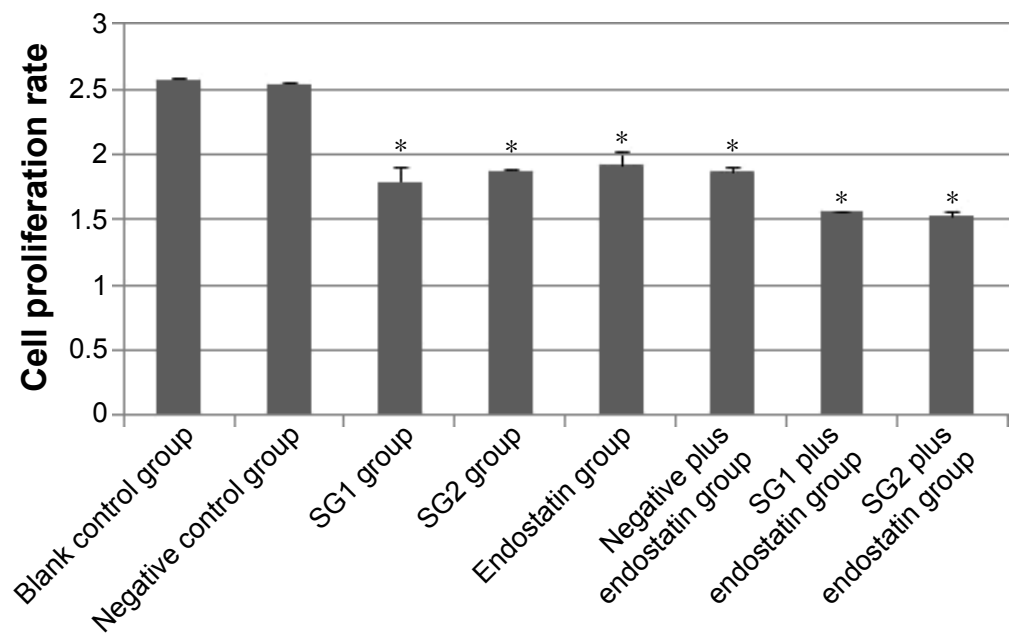

Figure 5 Proliferation of the KYSEI50 cells under the three-dimensional culture medium.

Notes: The cells were treated as described in "Materials and methods" section and then the cells were subjected to CCK 8 analysis. $* P<0.05$. 
and could better inhibit lymphatic vessel genesis. Thus, it could be speculated that both VEGF-C and endostatin played a significant role in the formation of esophageal cancer-related lymphatic vessels and the synergistic effect of both was stronger and obvious.

It would be better to co-culture the KYSE150 cells and lymphatic endothelial cells together. The cells could be fluorescently labeled red (endothelial cells) and green (KYSE150 cells). The endothelial cells would then be continuously exposed to the VEGF-C secreted by the KYSE150 cells (or its absence in small interfering VEGF-C cells). Endostatin could be added to the culture medium as needed for each group. VEGF-C should be added to the KYSE150 small interfering VEGF-C/endothelial cell cultures to see if ring formation can be rescued by the addition of VEGF-C.

The results are only shown by one cell line and must be confirmed in at least one additional esophageal squamous cell carcinoma cell line. All the data shown were in vitro. A limitation of this study is that the role of VEGF-C in primary esophageal squamous cell carcinomas was studied in vitro.

\section{Acknowledgment}

This work was supported by a Grant from the National Natural Science Foundation (81272370).

\section{Disclosure}

The authors report no conflicts of interest in this work.

\section{References}

1. Jennbacken K, Vallboc C, Wang W, Damber JE. Express ion of vascular endothelial growth factor $\mathrm{C}$ (VEGF-C) and VEGF receptor-3 in human prostate cancer is associated with regional lymph node metastasis. Prostate. 2005;65(2):110-116.

2. Juanjuan Ou, Peiliang Geng, Jianjun Li, et al. Inhibition effects of endostatin on EDA expression of colorectal carcinoma cells to affect formation of human lymph endothelial cells. Chin J Cancer Prev Treat. 2013;17(5):349-352.

3. Shang L, Zhao J, Wang W, et al. Inhibitory effect of endostar on lymphangiogenesis in non-small cell lung cancer and its effect on circulating tumor cells. Zhongguo Fei Ai Za Zhi. 2014;17(10):722-729.

4. Zhuo W, Chen Y, Song X, Luo Y. Endostatin specifically targets both tumor blood vessels and lymphatic vessels. Front Med. 2011;5(4): 336-340.

5. Hatva E, Kaipainen A, Mentula P, et al. Expression of endothelial cellspecific receptor tyrosine kinases and growth factors in human brain tumors. Am J Pathol. 1995;146(2):368-378.

6. Tianwen Xu, Daoda Chen, Jianying Chen, et al. The correlation of VEGF-C expression in colorectal carcinoma and its lymph node metastasis. Chin J Exp Surg. 2005;22(5):598-600.

7. Yunfeng Zhang, Ting Lei, Hengli Li, et al. Expression and significance of vascular endothelial growth factor-C in gallbladder carcinoma. Chin J Exp Surg. 2004;21(2):238.

8. Jun Ji, Chao Yan, Jun Zhang, et al. Expression of vascular endothelial growth factor $\mathrm{C}$ and its correlation with lymph node metastasis in gastric carcinoma. Chin J Exp Surg. 2004;21(5):530-532.

9. Kai Li, Jing Tao, Chunyou Wang. Relation between pancreatic cancer lymphatic vessel formation and vascular endothelial growth factor. Chin J Exp Surg. 2006;23(1):16-17.

10. Hirakawa S, Brown LF, Kodama S, Paavonen K, Alitalo K, Detmar M. VEGF-C induced lymphangiogenesis in sentinel lymph nodes promotes tumor metastasis to distant sites. Blood. 2007;109(3):1010-1017.

11. Fukumoto S, Morifuji M, Katakura Y, Ohishi M, Nakamura S. Endostatin inhibits lymph node metastasis by a down-regulation of the vascular endothelial growth factor $\mathrm{C}$ expression in tumor cells. Clin Exp Metastasis. 2005;22(1):31-38.

12. Brideau G, Makinen MJ, Elamaa H, et al. Endostatin overexpression inhibits lymphangiog-enesis and lymph node metastasis in mice. Cancer Res. 2007;67(24):11528-11535.
OncoTargets and Therapy

\section{Publish your work in this journal}

OncoTargets and Therapy is an international, peer-reviewed, open access journal focusing on the pathological basis of all cancers, potential targets for therapy and treatment protocols employed to improve the management of cancer patients. The journal also focuses on the impact of management programs and new therapeutic agents and protocols on

\section{Dovepress}

patient perspectives such as quality of life, adherence and satisfaction. The manuscript management system is completely online and includes a very quick and fair peer-review system, which is all easy to use. Visit http://www.dovepress.com/testimonials.php to read real quotes from published authors. 